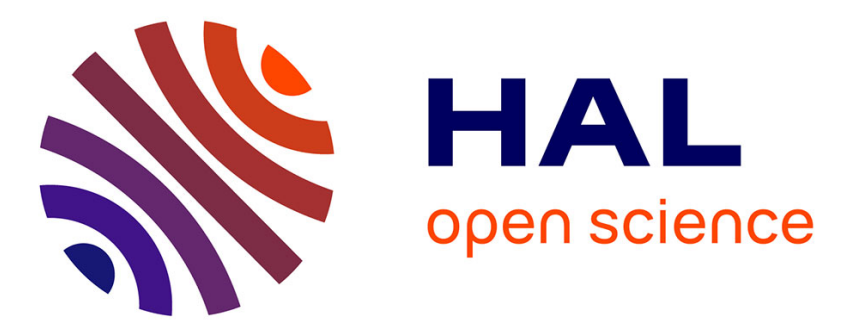

\title{
Analysis of an Experimental Model of In Vitro Cardiac Tissue Using Phase Space Reconstruction
}

\author{
Binbin Xu, Sabir Jacquir, Gabriel Laurent, Jean-Marie Bilbault, Stéphane
}

Binczak

\section{- To cite this version:}

Binbin Xu, Sabir Jacquir, Gabriel Laurent, Jean-Marie Bilbault, Stéphane Binczak. Analysis of an Experimental Model of In Vitro Cardiac Tissue Using Phase Space Reconstruction. Biomedical Signal Processing and Control, 2014, 10.1016/j.bspc.2014.06.005 . hal-01009125

\section{HAL Id: hal-01009125 \\ https://inria.hal.science/hal-01009125}

Submitted on 17 Jun 2014

HAL is a multi-disciplinary open access archive for the deposit and dissemination of scientific research documents, whether they are published or not. The documents may come from teaching and research institutions in France or abroad, or from public or private research centers.
L'archive ouverte pluridisciplinaire HAL, est destinée au dépôt et à la diffusion de documents scientifiques de niveau recherche, publiés ou non, émanant des établissements d'enseignement et de recherche français ou étrangers, des laboratoires publics ou privés. 


\title{
Analysis of an experimental model of in vitro cardiac tissue using phase space reconstruction
}

\author{
Binbin $\mathrm{Xu}^{1,2}$, Sabir Jacquir ${ }^{3 *}$, Gabriel Laurent ${ }^{3}$ Jean-Marie Bilbault ${ }^{3}$ Stéphane Binczak ${ }^{3}$, \\ 1 GEOSTAT, INRIA Bordeaux Sud-Ouest, Talence, France \\ 2 LIRYC, L'Institut de RYthmologie et modélisation Cardiaque, Bordeaux, France \\ 3 CNRS UMR 6306, LE2I Université de Bourgogne, Dijon France * \\ sjacquir@u-bourgogne.fr
}

\begin{abstract}
The in vitro cultures of cardiac cells represent valuable models to study the mechanism of the arrhythmias at the cellular level. But the dynamics of these experimental models cannot be characterized precisely, as they include a lot of parameters that depend on experimental conditions. This paper is devoted to the investigation of the dynamics of an in vitro model using a phase space reconstruction. Our model, based on the heart cells of new born rats, generates electrical field potentials acquired using a microelectrode technology, which are analyzed in normal and under external stimulation conditions. Phase space reconstructions of electrical field potential signals in normal and arrhythmic cases are performed after characterizing the nonlinearity of the model, computing the embedding dimension and the time lag. A non-parametric statistical test (Kruskal-Wallis test) shows that the time lag $\tau$ could be used as an indicator to detect arrhythmia, while the embedding dimension is not significantly different between the normal and the arrhythmia cases. The phase space reconstructions highlight attractors, whose dimension reveals that they are strange, depicting a deterministic dynamics of chaotic nature in our in vitro model.
\end{abstract}

\section{Introduction}

World Health Organization expects that the annual deaths due to cardiovascular diseases will increase from 17 million in 2008 to 25 million in 2030 in the world [1]. Among them, cardiac arrhythmias are known to be responsible for many cardiovascular deaths, while atrial fibrillation plays specifically a major role in arrhythmic disorders. Although there exists a rich body of literature studying cardiovascular diseases, a limiting factor is the poor availability of experimental models to reproduce arrhythmias, which could 
help to understand the triggering mechanisms at the cellular level.

In order to overcome the limitations of in vivo heart studies (availability, heart beating etc.), cultures from cardiac muscular cells were developed [2]. This kind of in vitro cardiac cultures keeps the general properties of the heart (electrophysiological, mechanical, etc.) and represents thus a promising experimental model for the studies of cardiac electrophysiology and arrhythmia. For example, it can be used to study the mechanism of action potential propagation phenomena in cardiac tissue and leads to study how and why disorders of the lethal rhythm take place, as the cardiac fibrillation, the reentry, etc. Moreover, the extracellular recording of electric activity of such cultures with the MEA (Multi-Electrodes Array or Micro-Electrodes Array) makes possible to monitor the contractile cardiac preparations for a longer time. In addition, applied to the cardiac cultures, the MEA technology has a better spatial resolution than the mapping procedure by fluorescence and is less invasive than the conventional electrophysiology methods (intracellular recording or by patch-clamp) $[3,4,5,6]$.)

In our previous works, we validated $[7,8,9]$ the use of MEA technology to study the electrical impulse propagation (extracellular field potential, EFP) in cardiomyocytes culture under normal conditions. Specifically, our preliminary results showed that it is possible to generate arrhythmias (spiral waves SW) in culture by electrical stimulations [10, 11$]$, in agreement with results in [12] observing that rapid stimulations could alter cardiac conduction and thus induce arrhythmia.

However, one question rises to be answered: Is the experimental model stable under electrical stimulations? Our objective is therefore to study the dynamics of the experimental model by characterizing acquired signals from cardiac cell cultures in normal conditions and in case of cardiac arrhythmia. In addition, a qualitative assessment of the robustness of the model to noise and to measurement error is provided.

The physiological signals, generally acquired as time series, have significant nonlinear characteristics that conventional analysis methods (Dominant frequency (DF) [13], amplitude analysis [14], Wavelet Transform (WT) [15]) often fail to identify. In fact, the behavior of biological systems depends on many parameters variations and becomes almost unpredictable. Methods from chaos theory and nonlinear dynamics give the possibility to study these special behaviors and are therefore suitable for physiological signal processing. Among them, the phase space reconstruction method is a valuable tool to study this kind of dynamical systems [16]. The phase space consists of a set of typical trajectories of the system, each point corresponding to a system state. It gives information such as the existence of attractors or 
limit cycles for selected parameters values.

In this paper, we will use phase space reconstruction to study the stability of the cardiomyocytes experimental model under electrical stimulations.

The paper is organized as follows. In section (2), we present the cardiomyocytes culture and we describe the electrical stimulation process to obtain arrhythmic phenomenon. In section (3), the nonlinearity of the experimental data is investigated, the reconstruction of phase space is discussed, including how to define and evaluate the corresponding parameters. Finally, the main conclusions are summarized in section (4).

\section{Materials}

\subsection{Extracellular field potentials}

The MEA can be used to record extracellular field potential (EFP) of cardiac cells which are grown directly on it (Fig. 1. More details of culture preparation of the cardiac cells of the newborn rats are in [2]). The region of interest of a typical MEA is about $700 \mu \mathrm{m}$ to $5 \mathrm{~mm}$ long. In this area, 60 electrodes are aligned in a matrix form with an inter-electrodes distance of $100 \mu \mathrm{m}$. The planar electrodes have a diameter of $30 \mu \mathrm{m}$. There are also eight pairs of electrodes devoted to the electrical external stimulation.

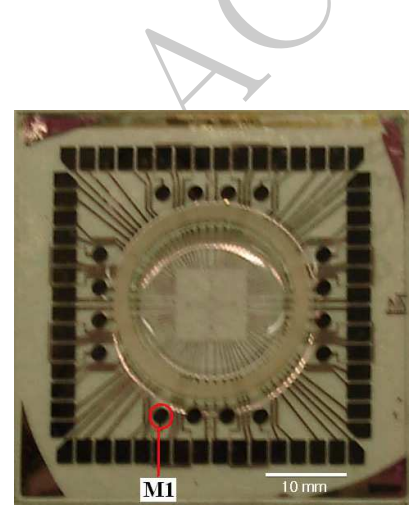

(a)

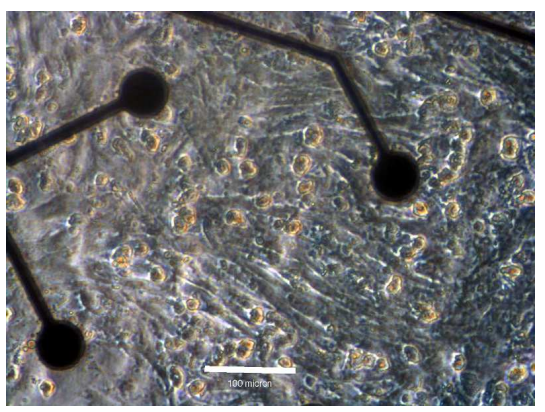

(b)

Figure 1. MEA with cardiac cellular culture of newborn rats. (a) MEA in vitro, stimulation electrode located at M1; (b) single-layer cardiomyocytes culture on the MEA, with $40 \times$ zoom.

The first experiments are carried out in normal conditions, which means that the cells are in the nutrient medium and are not stimulated (see signals from extracellular field potential in Fig. 2a). Each 
of $60 \mathrm{EFP}$ signals is acquired with a maximum sampling frequency of $50 \mathrm{kHz}$ per channel and a 12 bits resolution. The recorded data are then reconstructed as 2D activation maps to study the field potential, then placed according to their current position on the MEA $(8 \times 8$ form $)$.

\subsection{Arrhythmic signal generation by electrical stimulation}

Two methods are generally used to induce arrhythmic signals in heart or in cardiomyocytes culture: injection of specific drugs such as aconitine and acetylcholine [17] or by vagal or electrical stimulation [18]. In this work, the arrythmia has been caused by electrical stimulation. The isolated healthy heart is not very sensitive to the initiation of tachyarrhythmia and atrial fibrillation. Namely, the vulnerability to atrial fibrillation is reduced in the isolated heart or cardiac cultures, which is caused by the lack of activity of the parasympathetic system. Despite this limiting factor, rapid pacing (even with low energy levels) can alter cardiac conduction and induce arrhythmia $[12,19,20,21]$. In fact, the electrical stimulation can also be an efficient method of atrial defibrillation. In recent years, the concept of electrical stimulation or sub-threshold stimulation with low amplitude and high frequency became more and more known and accepted $[22,23,24]$. It would be interesting to test this concept on an experimental model, nevertheless, it requires to qualify the behavior of the experimental model under electrical stimulation.

The culture is stimulated by a pulse train (amplitude $200 \mu \mathrm{V}$, frequency $100 \mathrm{~Hz}$, excitation duration 5 min), sent by an electrode located at the edge of the MEA. The pacing is carefully chosen higher than the natural frequency $(1,5 \mathrm{~Hz})$ of the cardiac cell of the newborn rats, in order to disrupt its electrical

activities. This stimulation protocol impairs the electrical activity of cardiomyocytes represented by recorded irregular and disordered field potentials (see Fig. 3a).

\section{Results}

\subsection{Nonlinearity of EFP by surrogate data analysis}

Often, for phenomena involved in biological systems, biological or physiological time series exhibit nonlinear features, but, even if a signal contains nonlinear characters, its nonlinearity is not necessarily reflected in its measure. As a result, conventional linear methods may fail to characterize this signal. Nonlinear tools are more relevant in this case, for example those from chaos theory. Therefore, the first step consists of verifying the nonlinear signature to justify the use of nonlinear methods. 
One popular method to check the nonlinearity of a signal is surrogate data analysis (SDA) [25, 26]. Starting first from the target signal, a surrogate signal is generated with same key features (mean, variance and power spectra, etc.) as those extracted from the original signal. Then one performs linear tests for these surrogate data. In our case, we use the time-reversal asymmetry (TRA) method [27]. If comparing both signals gives any correlation, the linearity of the target signal is highlighted. In the opposite case (zero correlation), it is very likely that the target signal is nonlinear.

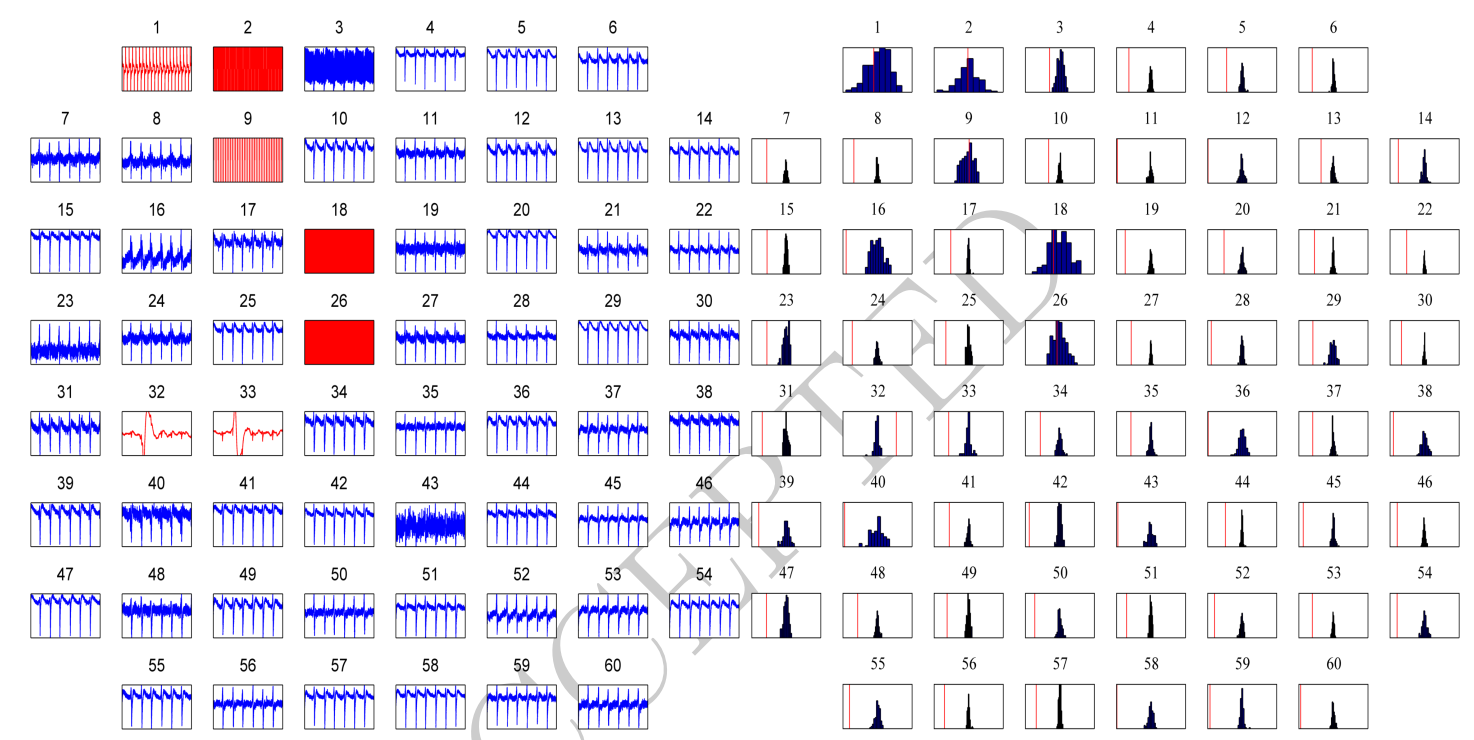

(a)

(b)

Figure 2. SDA test on normal EFP/signals. (a) original signals, (b) histogram of surrogate data, the vertical red line denoting the statistical value of the original signal.

Here, SDA is applied to EFP signals corresponding to two cases: normal signals (Fig. 2a) and arrhythmic signals (Fig. 3a). The normal case corresponds to the signals with a constant EFP frequency (for the data presented in the Fig. 2a, the frequency is equal to $1.5 \mathrm{~Hz}$ ). The arrhythmic case corresponds to the signals with a variable EFP frequency (Fig. 3a).

In Fig. 2b (resp. Fig. 3b), the dark blue distributions are histograms of SDA data for normal (resp. arrhythmic) case. The vertical red lines indicate the values of the TRA descriptor of the original signals. If the red lines drop in the distribution, original signal can be considered as linear. For example, recordings from electrodes providing periodic rectangular signals on one hand (identified by red color in Fig. 2a and Fig. 3a) are false signals, that is, they correspond to noisy signals, saturation of the amplificator, or a bad contact between cells and electrode and can be considered as linear signals. For normal extracellular 


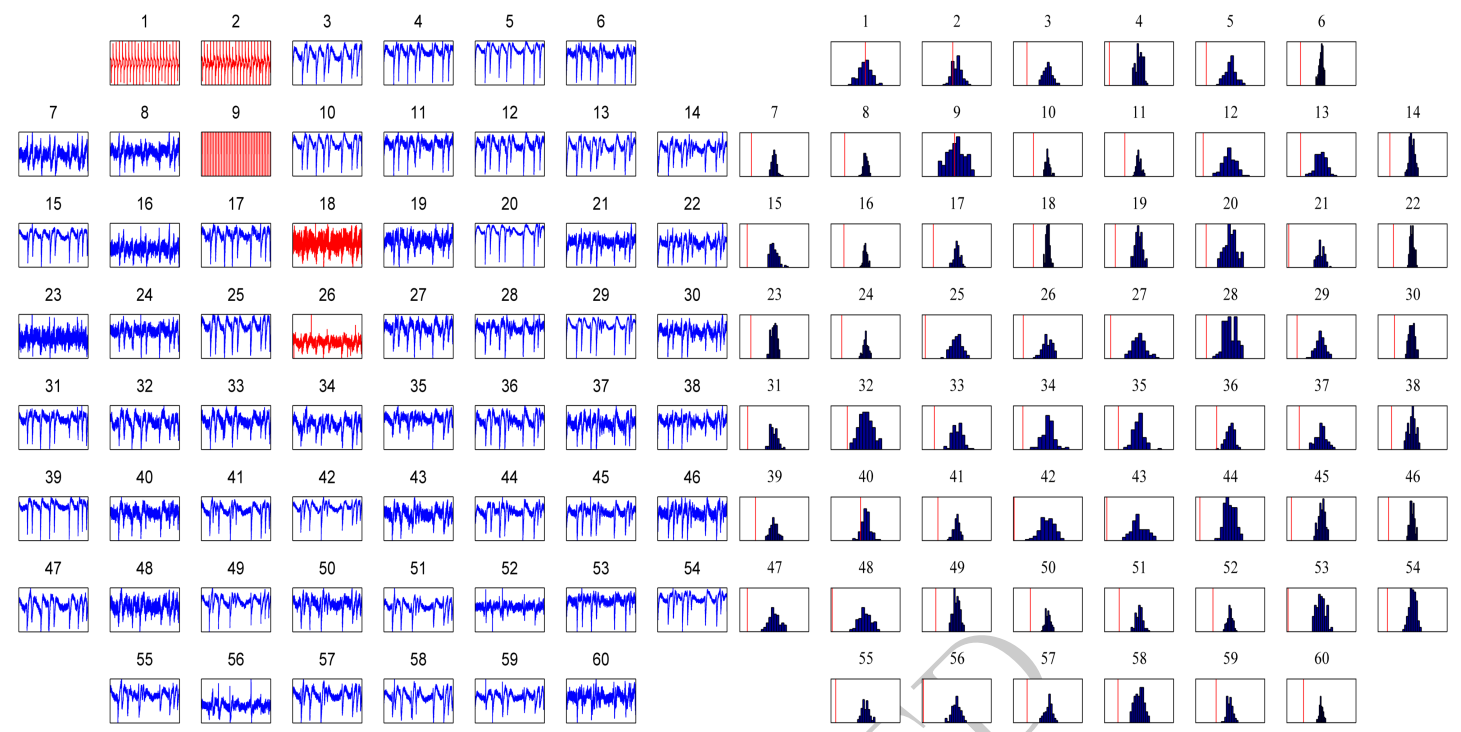

(a)

(b)

Figure 3. SDA test on arrhythmic EFP signals. (a) original signals, (b) histogram of surrogate data, the vertical red line denoting the statistical value of the original signal.

signals on the other hand, the red lines being almost all outside the linear distributions, their nonlinearity is depicted.

In general, nonlinearity of EFP signals is then confirmed except for the some false signals. All other acquired signals are marked by their nonlinearity. We will therefore use nonlinear methods to process these signals in the next section.

\subsection{Phase space reconstruction}

Having addressed the nonlinear feature of EFP signal, we now investigate the dynamic of the EFP signal using the phase space reconstruction method. The reconstructed space is characterized by only $m$ independent quantities representing the coordinates of a point in $m$-dimensional phase space. The challenge is to find the appropriate number $m$, such as the properties of the initial time series are kept in the reconstructed space. Theoretically, there are two possible methods of phase space reconstruction. The first one consists of differentiating the original signal with respect to time and considering $x(t), \frac{d x}{d t}, \frac{d^{2} x}{d t^{2}}, \ldots, \frac{d^{m-1} x}{d t^{m-1}}$. For numerical computation, the derivative calculation is too sensitive to differences and errors which depend on the different algorithms. The second method consists of building a $m$ dimensional system from a one dimensional time series with a fixed delay to shift the original data (time lagged method)[28]. In this 
case, the time lag $\tau$ must be evaluated. This second method does not require mathematically explicitly defined system, so it fits quite well with experimental data and one-dimensional time series. For these reasons, we choose this method and represent the system states with a normalized sample step $T_{s}=1$, $\tau \in \mathbb{N}^{*}$, while $p=0,1,2, . ., N$ are the indices of the succesive samples values of the time series :

$$
X(\tau, m)=[\vec{x}(p), \vec{x}(p+\tau), \vec{x}(p+2 \tau), \ldots, \vec{x}(p+(m-1) \tau)],
$$

where $X$ contains the phase space vectors:

- $\vec{x}(p)=\left(x(0), x(1), x(2), . ., x\left(N^{\prime}\right)\right)^{t}$

- $\vec{x}(p+\tau)=\left(x(\tau), x(1+\tau), x(2+\tau), . ., x\left(N^{\prime}+\tau\right)\right)^{t}$

- $\vec{x}(p+2 \tau)=\left(x(2 \tau), x(1+2 \tau), x(2+2 \tau), . ., x\left(N^{\prime}+2 \tau\right)\right)^{t}$

- $\vec{x}(p+(m-1) \tau)=\left(x((m-1) \tau), x(1+(m-1) \tau), x(2+(m-1) \tau), . ., x\left(N^{\prime}+(m-1) \tau\right)\right)^{t}$

The last coordinate of the phase space vector depends on the length of the signal, the time lag $\tau$ and the embedding dimension $m$. Namely, it corresponds to $N^{\prime}=N-(m-1) \tau$. Moreover, Takens theorem [28] gives conditions under which a nonlinear dynamical system can be reconstructed from a sequence of observation of its states. Both parameters $m$ and $\tau$ should be determined very carefully in order to build a correct phase space, as follows.

\subsubsection{Time lag $\tau$ :}

The parameter time lag $\tau$ influences the phase space reconstruction. If $\tau$ is too small, the trajectories of $\vec{x}(p)$ and $\vec{x}(p+\tau)$ are too close and can even be indistinguishable, creating redundant coordinates. On the other hand if $\tau$ is too large, the two coordinates are statistically independent. This implies that the trajectories of attractor projected on the two axes are not correlated, which makes the reconstruction phase space useless. The goal is to find the smallest value of $\tau$ so that the resulting coordinates are independent. Without an a priori knowledge of the system, especially for experimental data, it is not obvious to properly determine this parameter. The method of autocorrelation function (ACF) [29] is used here. The ACF is defined as

$$
C(\tau)=C(0) e^{-k \tau},
$$


where $C(0)$ is calculated without lag. $\tau$ is obtained when $C(\tau)=\frac{C(0)}{e}$, that means $k=\frac{1}{\tau}$. It corresponds to the time required for the autocorrelation function (ACF) to decrease to $1 / e$ of its original value [29]. By comparing results of $\tau$, as reported in Table 1, we find that the values of $\tau$ are higher for arrhythmic signals than for normal signals (Fig. 5). The mean time lag for normal signals is $44 \pm 10 \mathrm{~ms}$. When arrhythmia happens, $\tau$ increases to $78 \pm 16$ ms. A Kruskal-Wallis test has been performed in order to show that the parameter $\tau$ may serve as an indication of arrhythmia (Fig. 4). As the $p-$ value for the time lag being equal to $6.0210^{-18}$, we can conclude that the time lag is significantly higher for arrhythmic signals than for normal signals.

\begin{tabular}{lcc}
\hline \hline Electrode numbers & Normal EFP signals & Arrhythmic EFP signals \\
\hline 4 & 34 & 70 \\
5 & 48 & 76 \\
14 & 46 & 80 \\
15 & 40 & 70 \\
20 & 50 & 76 \\
29 & 54 & 78 \\
\hline
\end{tabular}

Table 1. $\tau$ values expressed in ms obtained by ACF for EFP signals. 6 electrodes have been randomly chosen as examples.

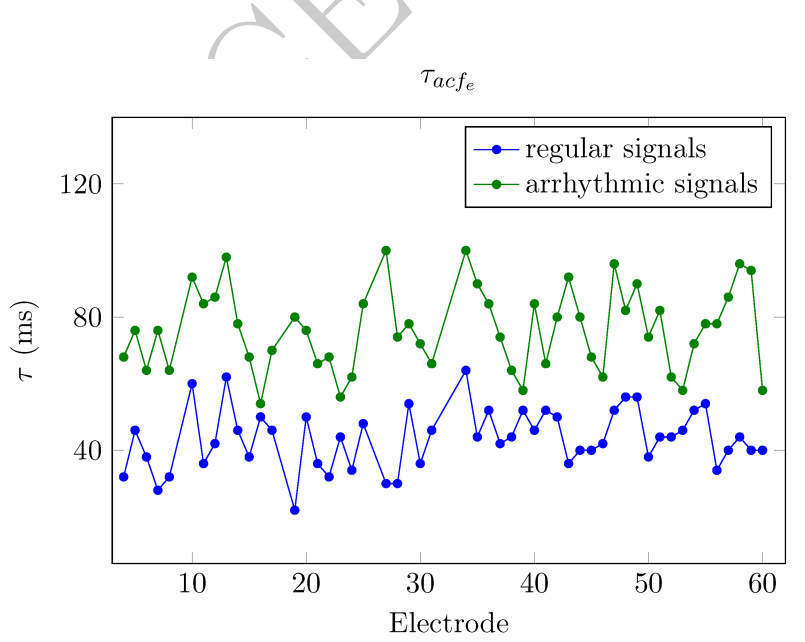

Figure 4. Comparison of $\tau$ for two types of EFP signals: Normal and arrhythmic.

\subsubsection{Embedding dimension $m$ :}

As previously mentioned, a well-defined embedding dimension is essential to a phase space in order to temporally describe all possible states of a dynamic system. For experimental data, the dimension of 


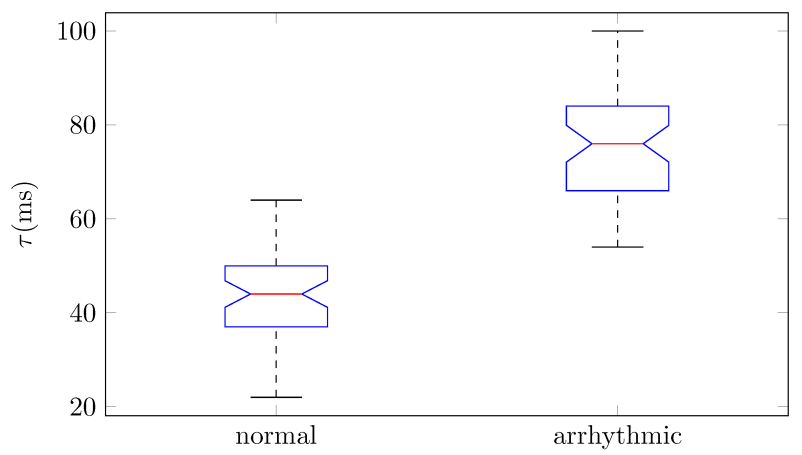

Figure 5. Distribution of $\tau$ for normal and arrhythmic EFP signals $\left(p=6.0210^{-18}\right.$, Kruskal-Wallis test with $n=52$ ).

the studied system is not known a priori. The aim is to ensure that the reconstructed space may well represent the original one, that is : both must be topologically identical. The phase space reconstruction is based on the principle of connecting the state points to create attractor trajectories of the system. If $m$ is not large enough, the state points on the trajectories could be very close, and there will be intersections between the trajectories of the attractor. The reconstructed space is then totally different from the original one. A higher value of $m$, theoretically, is possible by just creating a larger space that contains minimal space. However, this will not only cause redundant problems, but also be an inefficiency issue, as it requires important computation power.

The most common method used to estimate the embedding dimension is the False Nearest Neighbor (FNN) [30], where the best value of $m$ corresponds to the minimum value of $m$ for which the FNN is close to zero. From a geometrical point of view, the time series (experimental measurements) is the projection of a $m$ dimensional system to a one-dimensional space. Therefore, two points in $m$ dimension space, even far from each other, could be very close ( and even be superimposed) in original 1D space. These points are called false neighbors. Results examples for both cases (normal and arrhythmic), corresponding to the experimental data presented in this paper, are shown in Table 2. A statistical test (Kruskal-Wallis

\begin{tabular}{lcc}
\hline \hline Electrode numbers & Normal EFP signals & Arrhythmic EFP signals \\
\hline 4 & 5 & 5 \\
5 & 4 & 7 \\
14 & 4 & 3 \\
15 & 4 & 5 \\
20 & 4 & 5 \\
29 & 5 & 4 \\
\hline
\end{tabular}

Table 2. Embedding dimension $m$ obtained by FNN, for the same electrodes as in Table 1. 


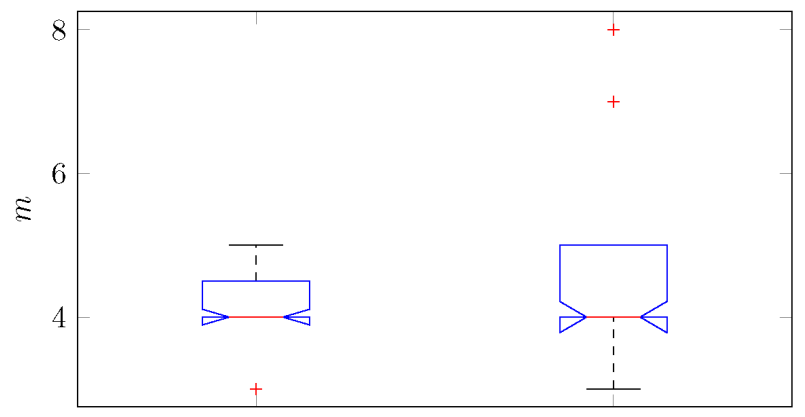

normal arrhythmic

Figure 6. Distribution of $m$ for normal and arrhythmic EFP signals ( $p=0.9651$, Kruskal-Wallis test with $n=52$ ).

test) has been performed in order to show that there is no significant difference between the embedding dimension of normal and arrhythmic signals (Fig. 6). The $p$-value for the embedding dimension being equal to $0.9651(p>0.001)$, we can conclude that there is no significant difference between the embedding dimension of normal signals and arrhythmic signals. The values of $m$, reported by our study, are in the range of embedding dimensions reported by other studies for cardiac signals [31, 32, 33, 34]. Even though it is often necessary to use high-dimensional numerical models to represent the cardiac cell / tissue, the global dynamics seem to remain low-dimensional. This happens for many biological / physiological systems, as for heart rate variability [35], electroencephalogram (EEG) [36] etc.

\subsubsection{Phase space reconstruction}

Using the parameters $m$ and $\tau$ computed previously, the phase space is reconstructed for the electrodes taken as examples. Although the embedded dimensions are greater than 3, for graphical convenience, we present only $2 D$ and $3 D$ projections (Fig. 7 and 8) for normal and for arrhythmic signals (Fig. 9 and 10).

The trajectories of the normal signals illustrate the existence of attractors in their phase spaces. The global forms of these trajectories are rather triangular, the widths of the trajectories being compact, which means that the correlation between the trajectories is strong (Fig. 7 and Fig. 8). In case of arrhythmias (Fig. 9 and Fig. 10), the reconstructed phase space becomes more disturbed and very scattered. We can also note that the arrhythmic signal's attractors are more scattered. The time lag of arrhythmic signals (resp. normal signals) has also been used to reconstruct the phase space of normal signals (resp. arrhythmic signals). We remarked that the attractor shapes remain globally similar, meaning that the time lag $\tau$ does not affect the dynamics of the model. 


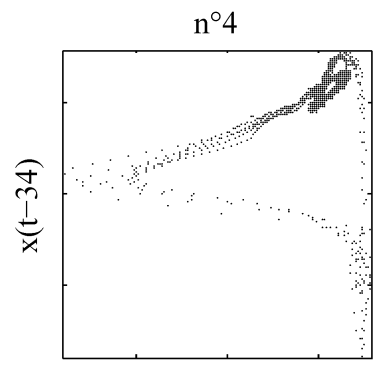

$\mathrm{x}(\mathrm{t})$

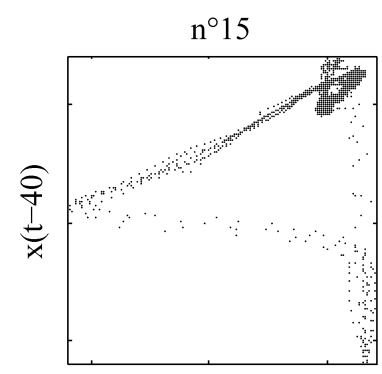

$\mathrm{x}(\mathrm{t})$

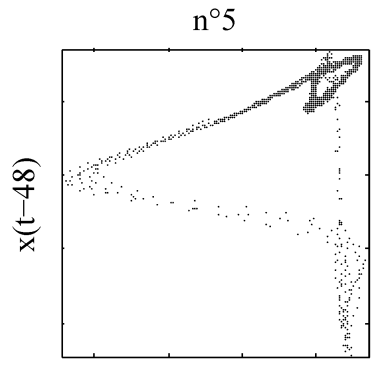

$\mathrm{x}(\mathrm{t})$

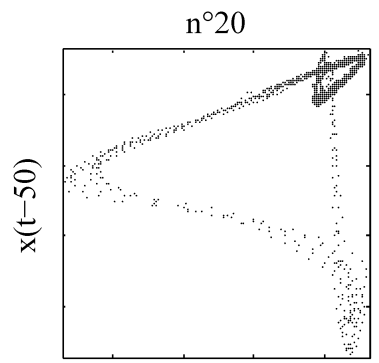

$\mathrm{x}(\mathrm{t})$

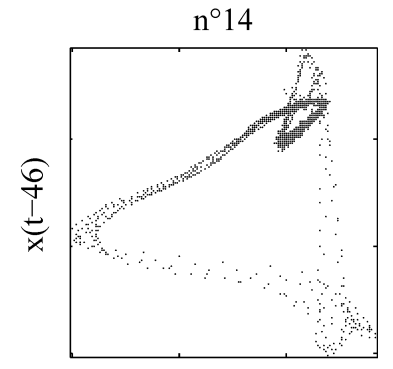

$x(t)$

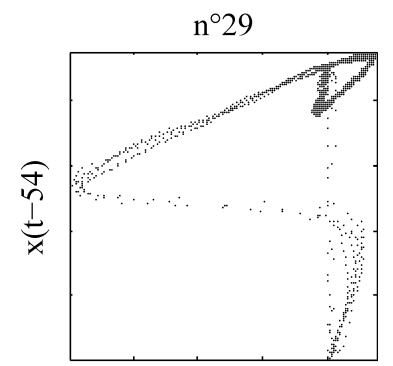

$x(t)$

Figure 7. Examples of 2D projection of the phase space reconstruction for normal signals. The electrode numbers are 4, 5, 14, 15, 2029.

In addition, if one wants to compare the attractors in the phase space reconstruction, then it would be worthwhile that the parameters used $(m$ and $\tau$ ) to reconstruct the arrhythmic and normal signals be consistent. For instance, one can reconstruct the phase space using the same parameters for all signals in order to demonstrate if there are any significant differences between attractors of arrhythmic and normal signals. From the box plots, the mean value of $m$ for both groups are roughly the same (around 4 ) while the mean value of $\tau$ is approximately 45 for normal signal and 75 for arrhythmic signals. The phase space reconstruction have been realized in two cases $(m=4, \tau=45$ and $m=4, \tau=75)$, as illustrated in Fig.11 for normal signals and Fig.12 for arrhythmic signals. In the two reconstructions (for normal and for arrhythmic signals), we remarked that the attractor shapes remain globally similar, meaning that the time lag does not affect the dynamics of the model. We can also note that for normal signals, the global forms of the trajectories are rather triangular, the widths of the trajectories are compact. In case of arrhythmias, the reconstructed phase space becomes more disturbed and very scattered, the attractors are also more scattered.

In the following section, we characterize the attractor features using the correlation dimension. 

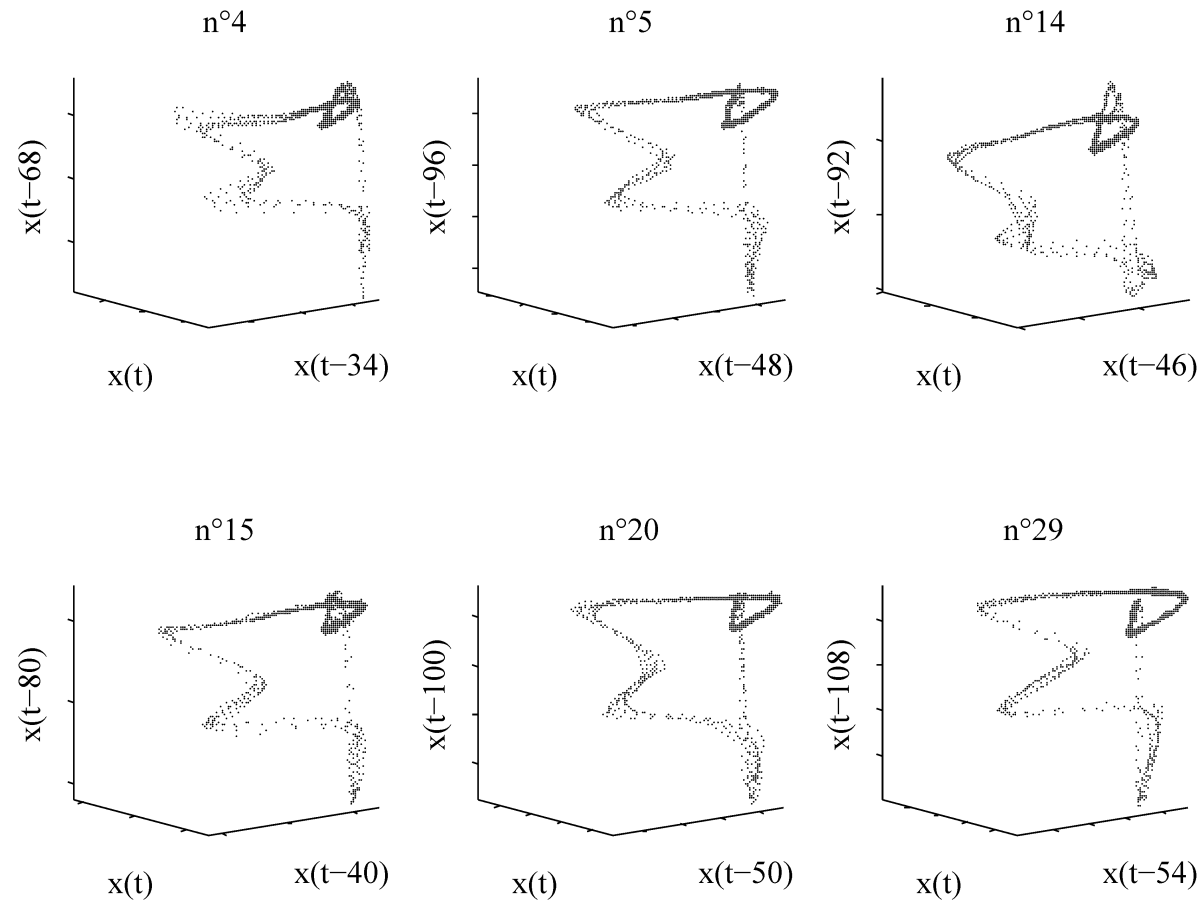

Figure 8. Examples of 3D projection of the phase space reconstruction for normal signals. The electrode numbers are 4, 5, 14, 15, 20, 29.

\subsubsection{Characterization of attractors}

The correlation dimension $D$ measures the geometrical complexity of an attractor [37]. It is associated with the organisation of points in phase space. In general, a strange attractor is characterized by a finite non-integer correlation dimension. More complex dynamics will result in a larger value of the correlation dimension. If the dynamics is stochastic, $D$ tends to infinity. An alternative worthwhile interpretation mentioned in [37] is that the correlation dimension gives the number of state variables needed to describe the dynamics around the attractor with a deterministic model.

Here, the correlation dimension is determined using the Grassberger-Procaccia (GP) method [38], based on the following approximation : the probability of having a couple of points in a box of size $r$ is equal to the probability of having a couple of points with separation distance less than $r$ when $r \longrightarrow 0$. The correlation dimension is defined by :

$$
D=\lim _{r \longrightarrow 0}\left[\frac{\log \left(C_{m}(r)\right)}{\log (r)}\right],
$$




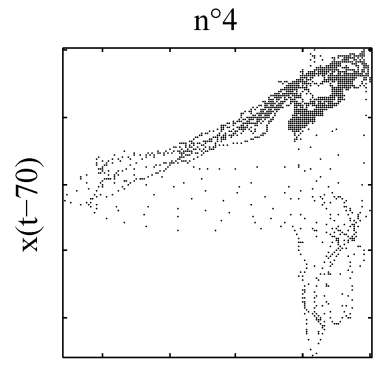

$\mathrm{x}(\mathrm{t})$

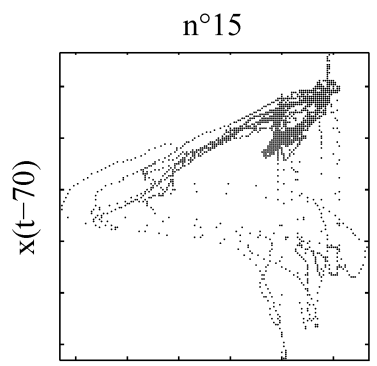

$x(t)$

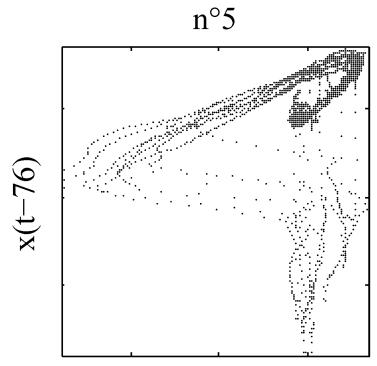

$x(t)$

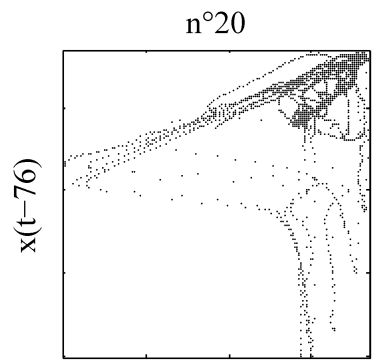

$x(t)$

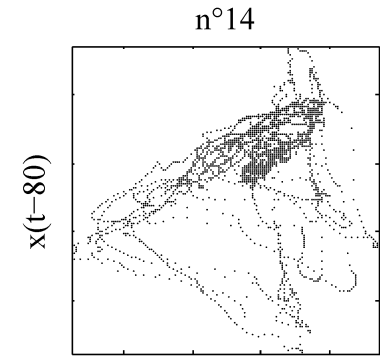

$\mathrm{x}(\mathrm{t})$

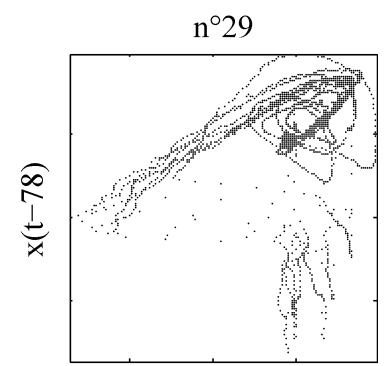

$x(t)$

Figure 9. Examples of $2 \mathrm{D}$ projection of the phase space reconstruction for arrhythmic signals. The electrode numbers are 4, 5,14,15,20, 29.

where the correlation integral $C_{m}(r)$ is approximately given by:

$$
C_{m}(r) \approx \frac{2}{N(N-1)} \sum_{p=1, i>p}^{N} \Theta\left(r-\left\|x_{p}-x_{i}\right\|\right),
$$

where $\Theta(x)$ is the Heaviside step function. The summation counts the number of pairs $\left(x_{p}, x_{i}\right)$ for which the distance $\left\|x_{p}-x_{i}\right\|$ is less than $r$. For each reconstructed phase space trajectory (Figs. 7 and 9), the distances between all points in the trajectory are calculated and the logarithm of the smallest distance (represented by $r_{\min }$ ) and the logarithm of the largest distance (represented by $r_{\max }$ ) are computed. A series of bins is then created to record the correlation sum, $C_{m}(r)$, which is the normalized number of couples of points with a separation distance less than a specified distance $r$. The process of depositing counts of data into bins is analogous to recording counts of the occurrence of events within data in a frequency histogram. In this study, an arbitrary number of 32 bins is used and the width of each bin is set to $\left(r_{\max }-r_{\min }\right) / 32$. Thus, from first to last, the separation distances $r_{n}=r_{\min }+n\left(r_{\max }-r_{\min }\right) / 32$, where $n=1$ to 32 are considered. In practice, the correlation dimension is obtained from the slope of 


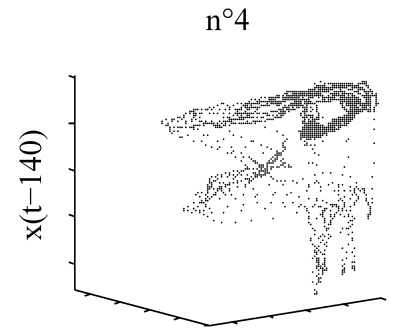

$x(t)$

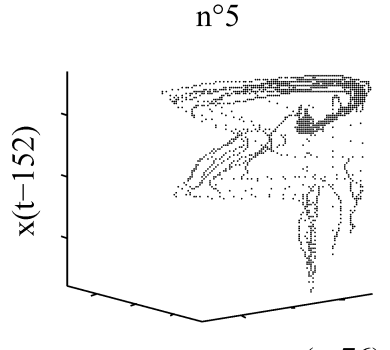

$x(t)$ $x(t-76)$

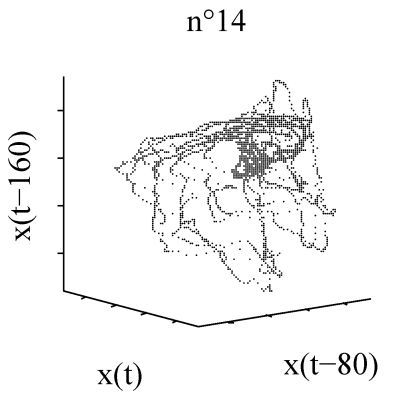

$n^{\circ} 15$

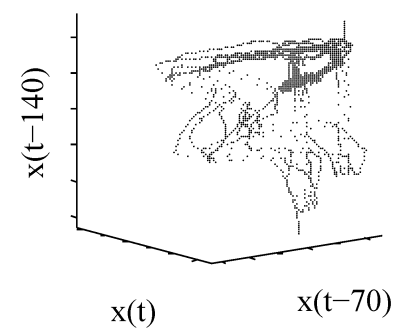

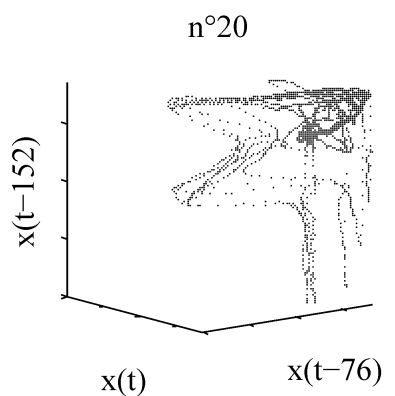

$x(t)$

$x(t-76)$

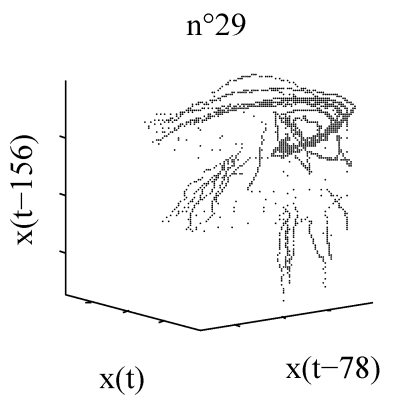

Figure 10. Examples of $3 \mathrm{D}$ projection of the phase space reconstruction for arrhythmic signals. The electrode numbers are 4, 5, 14, 15, 20, 29.

$\log \left(C_{m}(r)\right)$ versus $\log (r)$. Several $C_{m}(r)$ are computed for increasing values of the embedding dimension $m$, and the slopes are determined from a scaling region of the log-log plot, as shown in Fig. 13 and Fig. 14 (see [38] for the scaling region determination). As shown by [39, 40], when $m$ increases, $D$ tends to a constant value of saturation corresponding to the correlation dimension (Figs. 15 and 16). For the data presented in this paper, a statistical test (Kruskal-Wallis test) (Fig.17) shows that there is a statistically significant difference between normal and arrhythmic cases $\left(p=2.774210^{-4}\right)$. The D values obtained from the EFP signals indicate that the attractors are strange. That means also the in vitro culture of cardiac cells performs in a low-dimensional chaos $(D<5)[37]$.

\subsubsection{Fractal dimension}

The complexity of time series is quantified by computing a fractal dimension $d$. Among the fractal dimension estimators in the litterature, the results given by three methods are presented in this paper: 


\section{Fractal dimension by Boxcount estimator}

Box-count estimator [41] is the most popular method to estimate fractal dimension. Suppose that the original time series graph is fully covered by a box. Then this box is divided into four sub-boxes for example. The number of boxes needed to cover the whole time series is counted. Following the same rule, these sub-boxes are divided into smaller boxes. The different box sizes $\epsilon$ (or called scale) and the number $N$ of validated boxes are recorded. The slope of the curve $\log N(\epsilon)$ versus $\log \epsilon$ gives the fractal dimension.

$$
\mathrm{FD}_{b o x}=\lim _{\epsilon \rightarrow 0} \frac{\log N(\epsilon)}{\log (1 / \epsilon)} .
$$

\section{Fractal dimension by Hall-Wood estimator}

In 1993, Hall and Wood found that, if the time series is modeled by a Gaussian process, concise formula may be developed for asymptotic bias and variance of box-counting estimators [42]. This gives a new version of fractal dimension by box-counting. It operates on the smallest $\epsilon$. Instead of estimating the slope of $\log N(\epsilon)$ versus $\log \epsilon, \mathrm{FD}_{h w}$ used another parameter $A(\epsilon)$. It denotes the total area of the recovered boxes at scale $\epsilon$ and $A(\epsilon) \propto N(\epsilon) \epsilon^{2}$. So,

$$
\mathrm{FD}_{h w}=2-\lim _{\epsilon \rightarrow 0} \frac{\log A(\epsilon)}{\log \epsilon}
$$

\section{Fractal dimension by Variation estimator}

A way to estimate the fractal dimension is to compute a "variation estimator" which is based on the 2nd order variogram of a stochastic process $[43,41]$.

As shown in Fig.18, Fig.19 and Fig.20, the normal EFP signals have a smaller fractal dimension than arrhythmic ones. This means that graphs from normal EFP signals are more likely to be smooth. Our results show also that Fractal dimension could also be used as a classification feature and it is an interesting result. 


\section{Conclusion}

In our previous works, cardiac arrhythmias have been performed using an electrical stimulation [11, 44]. Starting from the presence of chaos in the bifurcation diagram for the periods of EFP signals [11], in the current work, the main objective is the characterization of EFP signals using nonlinear features. The comparison of two specific cases (normal and arrhythmic ones) has been performed in order to verify that the embedded dimension was robust against perturbation and to study its effect on other parameters such as the time delay. Our study shows that the time delay can be used as a feature to classify arrhythmic and normal signal, while the embedding dimension remains unchanged and therefore cannot be used for classification purposes. The phase space reconstruction has shown that the dynamics of the model present significant behavioral changes between normal and arrhythmic cases. Furthermore, for each case, all the electrodes have given the same qualitative results, even if intrinsic and extrinsic noise is present. The nonlinearity of the model is confirmed by surrogate data analysis for normal and for arrhythmic cases. In a phase space, the arrhythmic signals are marked by strongly disturbed trajectories, unlike those in the normal case that are regular. However, the global dynamics of the arrhythmic signals remains unchanged, which is reflected by attractors in phase space. The values of the correlation dimension qualify the attractors as strange. The statistical test of the fractal dimension shows a difference between the normal and arrhythmic EFP signals. The fractal dimension has been computed using three methods

(Box-count, Hall-Wood and Variogram). We should emphasize the significance of this result, showing that our in vitro model underscores a deterministic chaotic dynamics. In summary, the external electrical stimulation is used to perturb the system but not sufficiently to modify its dimension. Nevertheless, it affects the temporal dynamics of the electrical activity of the tissue. Therefore, it leads to a change of time delay as the frequencies of the signals are modified. We can conclude that the external perturbations imply only a change of trajectories in the same embedded space. The experimental model based on the MEA technology provides a robust tool for studying the mechanism of cardiac arrhythmia and the defibrillation by sub-threshold electrical stimulation [45]. Finally, the phase space reconstruction is a useful tool to study the dynamics of time series given by an experimental model, and may further be used for classification purposes [16]. It could also allow the asymptotic identification of state variables of a theoretical model knowing an a priori embedding dimension and a time lag. 


\section{Acknowledgements}

B. Xu is financially supported by the French IHU in cardiac rhythmology L'Institut de rythmologie et modélisation cardiaque LIRYC and the regional council of Aquitaine. The authors would like to thank also the Council of Burgundy (Dijon, France) for financial support.

\section{References}

\section{References}

1. W. H. Organization (Ed.), World Health Statistics 2012, World Health Organization, 2012.

2. P. Athias, D. Vandroux, C. Tissier, L. Rochette, Development of cardiac physiopathological models from cultured cardiomyocytes, Annales de Cardiologie et d'Angéiologie 55 (2) (2006) 90-99.

3. F. Pillekamp, M. Reppel, K. Brockmeier, J. Hescheler, Impulse propagation in late-stage embryonic and neonatal murine ventricular slices, Journal of Electrocardiology 39 (4) (2006) 425.e1-425.e4.

4. A. Stett, U. Egert, E. Guenther, F. Hofmann, T. Meyer, W. Nisch, H. Haemmerle, Biological application of microelectrode arrays in drug discovery and basic research, Analytical and Bioanalytical Chemistry 377 (3) (2003-10-12) 486-495.

5. K. Banach, M. D. Halbach, P. Hu, J. Hescheler, U. Egert, Development of electrical activity in cardiac myocyte aggregates derived from mouse embryonic stem cells, American Journal of Physiology - Heart and Circulatory Physiology 284 (6) (2003) H2114-H2123.

6. J. Hescheler, M. Halbach, U. Egert, Z. J. Lu, H. Bohlen, B. K. Fleischmann, M. Reppel, Determination of electrical properties of es cell-derived cardiomyocytes using meas, Journal of Electrocardiology 37 (Supplement 1) (2004) 110-116.

7. G. Laurent, S. Jacquir, S. Binczak, D. Vandroux, O. Bouchot, J. Wolf, P. Athias, J. Bilbault, Establishing a novel in vitro model for the study of spiral waves during arrhythmia, European Heart Journal 29 (2008) 166. 
8. P. Athias, S. Jacquir, C. Tissier, D. Vandroux, S. Binczak, J. Bilbault, M. Rossé, Excitation spread in cardiac myocyte cultures using paired microelectrode and microelectrode array recordings, Journal of Molecular and Cellular Cardiology 42 (6) (2007) S3-S3.

9. S. Jacquir, C. Tissier, D. Vandroux, S. Binczak, J. Bilbault, M. Rossé, P. Athias, Paired microelectrodes and microelectrode array analysis of cardiac impulse propagation in cardiomyocyte cultures, Fundamental Clinical Pharmacology 22 (1) (2008) 51-52.

10. P. Athias, S. Jacquir, G. Laurent, D. Vandroux, S. Binczak, J. Bilbault, In vitro simulation of spiral waves in cardiomyocyte networks using multi-electrode array technology, European Journal of Heart Failure Supplements 8 (2009) 963.

11. S. Jacquir, S. Binczak, B. Xu, G. Laurent, D. Vandroux, P. Athias, J. Bilbault, Investigation of micro spiral waves at cellular level using a microelectrode array technology, Int. J. Bifurcation Chaos 21 (2011) 1-15.

12. A. A. Kondratyev, J. G. C. Ponard, A. Munteanu, S. Rohr, J. P. Kucera, Dynamic changes of cardiac conduction during rapid pacing, American Journal of Physiology - Heart and Circulatory Physiology 292 (4) (2007) H1796-H1811.

13. S. M. Narayan, M. Wright, N. Derval, A. Jadidi, A. Forclaz, I. Nault, S. Miyazaki, F. Sacher, P. Bordachar, J. Clmenty, P. Jas, M. Hassaguerre, M. Hocini, Classifying fractionated electrograms in human atrial fibrillation using monophasic action potentials and activation mapping: Evidence for localized drivers, rate acceleration, and nonlocal signal etiologies, Heart Rhythm 8 (2) (2011) $244-253$.

14. M. A. WOOD, P. MOSKOVLJEVIC, B. S. STAMBLER, K. A. ELLENBOGEN, Comparison of bipolar atrial electrogram amplitude in sinus rhythm, atrial fibrillation, and atrial flutter, Pacing and Clinical Electrophysiology 19 (2) (1996) 150-156.

15. R. Houben, N. de Groot, M. Allessie, Analysis of fractionated atrial fibrillation electrograms by wavelet decomposition, Biomedical Engineering, IEEE Transactions on 57 (6) (2010) 1388-1398.

16. M. Chen, Y. Fang, X. Zheng, Phase space reconstruction for improving the classification of single trial eeg, Biomedical Signal Processing and Control 11 (0) (2014) 10-16. 
17. M. Allessie, W. Lammers, F. Bonke, J. Hollen, Experimental evaluation of moe's multiple wavelet hypothesis of atrial fibrillation, Cardiac Electrophysiology and Arrhythmias. New York: Grune \& Stratton (1985) 265-276.

18. Y. Yeh, K. Lemola, S. Nattel, Vagal atrial fibrillation, Acta Cardiologica Sinica 23 (1) (2007) 1-12.

19. M. Allessie, C. Kirchhof, G. Scheffer, F. Chorro, J. Brugada, Regional control of atrial fibrillation by rapid pacing in conscious dogs, Circulation 84 (4) (1991) 1689-1697.

20. G. Laurent, G. Moe, X. Hu, H. Leong-Poi, K. A. Connelly, P. P.-S. So, A. Ramadeen, L. Doumanovskaia, A. Konig, J. Trogadis, D. Courtman, B. Strauss, P. Dorian, Experimental studies of atrial fibrillation: a comparison of two pacing models, American Journal of Physiology - Heart and Circulatory Physiology 294 (3) (2008) H1206-H1215.

21. G. Laurent, G. W. Moe, X. Hu, P. Pui-Sze So, A. Ramadeen, H. Leong-Poi, L. Doumanovskaia, A. Konig, J. Trogadis, D. Courtman, B. H. Strauss, P. Dorian, Simultaneous right atrioventricular pacing: A novel model to study atrial remodeling and fibrillation in the setting of heart failure, Journal of Cardiac Failure 14 (3) (2008) 254-262.

22. G. Osipov, B. Shulgin, J. Collins, Controlled movement and suppression of spiral waves in excitable media, Phys. Rev. E 58 (6) (1998) 6955-6958.

23. H. Zhang, B. Hu, G. Hu, Suppression of spiral waves and spatiotemporal chaos by generating target waves in excitable media, Phys. Rev. E 68 (2) (2003) 26134.

24. T. K. Shajahan, A. R. Nayak, R. Pandit, Spiral-wave turbulence and its control in the presence of inhomogeneities in four mathematical models of cardiac tissue, PLoS ONE 4 (3) (2009) 4738.

25. T. Schreiber, A. Schmitz, Improved surrogate data for nonlinearity tests, Phys. Rev. Lett. 77 (4) (1996) 635-638.

26. J. Mazaraki, Dynamical methods for analysing and forecasting chaotic data, Honours thesis, University of New South Wales (1997).

27. T. Schreiber, A. Schmitz, Discrimination power of measures for nonlinearity in a time series, Phys. Rev. E 55 (5) (1997) 5443-. 
28. F. Takens, Detecting strange attractors in turbulence, in: D. Rand, L.-S. Young (Eds.), Dynamical Systems and Turbulence, Lecture Notes in Mathematics, Vol. 898, Springer Berlin / Heidelberg, 1981, pp. 366-381.

29. A. M. Albano, J. Muench, C. Schwartz, A. I. Mees, P. E. Rapp, Singular-value decomposition and the grassberger-procaccia algorithm, Phys. Rev. A 38 (6) (1988) 3017-3026.

30. M. B. Kennel, R. Brown, H. D. I. Abarbanel, Determining embedding dimension for phase-space reconstruction using a geometrical construction, Phys. Rev. A 45 (6) (1992) 3403-3411.

31. J. Fell, K. Mann, J. Röschke, M. S. Gopinathan, Nonlinear analysis of continuous ECG during sleep I. Reconstruction, Vol. 82, Springer-Verlag, 2000, pp. 477-483.

32. J. Fell, K. Mann, J. Röschke, M. S. Gopinathan, Nonlinear analysis of continuous ECG during sleep II. Dynamical measures, Vol. 82, Springer-Verlag, 2000, pp. 485-491.

33. K. Antanavičius, A. Bastys, J. Blužas, L. Gargasas, S. Kaminskienė, G. Urbonavičienè, A. Vainoras, Nonlinear dynamics analysis of electrocardiograms for detection of coronary artery disease, Computer Methods and Programs in Biomedicine 92 (2) (2008) 198-204.

34. O. Mironyuk, A. Loskutov, Detection of cardiac pathologies using dimensional characteristics of rr intervals in electrocardiograms 51 (1) (2006) 115-119.

35. J.-F. Casties, D. Mottet, D. Le Gallais, Non-linear analyses of heart rate variability during heavy exercise and recovery in cyclists., Int J Sports Med 27 (10) (2006) 780-785.

36. A. Celletti, A. Villa, Low-dimensional chaotic attractors in the rat brain, Vol. 74, Springer-Verlag, 1996, pp. 387-393.

37. B. Hoekstra, C. Diks, M. Allessie, J. DeGoede, Nonlinear analysis of epicardial atrial electrograms of electrically induced atrial fibrillation in man, Journal of Cardiovascular Electrophysiology 6 (1995) 419-440.

38. P. Grassberger, I. Procaccia, Characterization of strange attractors, Phys. Rev. Lett. 50 (5) (1983) 346-349. 
39. M. Ding, C. Grebogi, E. Ott, T. Sauer, J. A. Yorke, Plateau onset for correlation dimension: When does it occur?, Phys. Rev. Lett. 70 (25) (1993) 3872-3875.

40. M. Ding, C. Grebogi, E. Ott, T. Sauer, J. A. Yorke, Estimating correlation dimension from a chaotic time series: when does plateau onset occur?, Physica D: Nonlinear Phenomena 69 (3-4) (1993) 404-424.

41. T. Gneiting, H. Sevcikova, D. B. Percival, Estimators of fractal dimension: Assessing the roughness of time series and spatial data, Statistical Science 27 (2) (2012) 247-277.

42. P. Hall, A. Wood, On the performance of box-counting estimators of fractal dimension, Biometrika 80 (1) (1993) 246-251.

43. R. J. Adler, Sample function erraticism and hausdorff dimension, in: The Geometry of Random Fields, John Wiley \& Sons Ltd., 1981, Ch. 8, pp. 184-252.

44. B. Xu, S. Jacquir, G. Laurent, J. Bilbault, S. Binczak, Spiral wave induced numerically using electrical stimulation and comparison with experimental results, in: Engineering in Medicine and Biology Society (EMBC), 2010 Annual International Conference of the IEEE, 2010, pp. 2650 -2653.

45. B. Xu, S. Jacquir, G. Laurent, J.-M. Bilbault, S. Binczak, A hybrid stimulation strategy for suppression of spiral waves in cardiac tissue, Chaos, Solitons \& Fractals 44 (8) (2011) 633-639. 

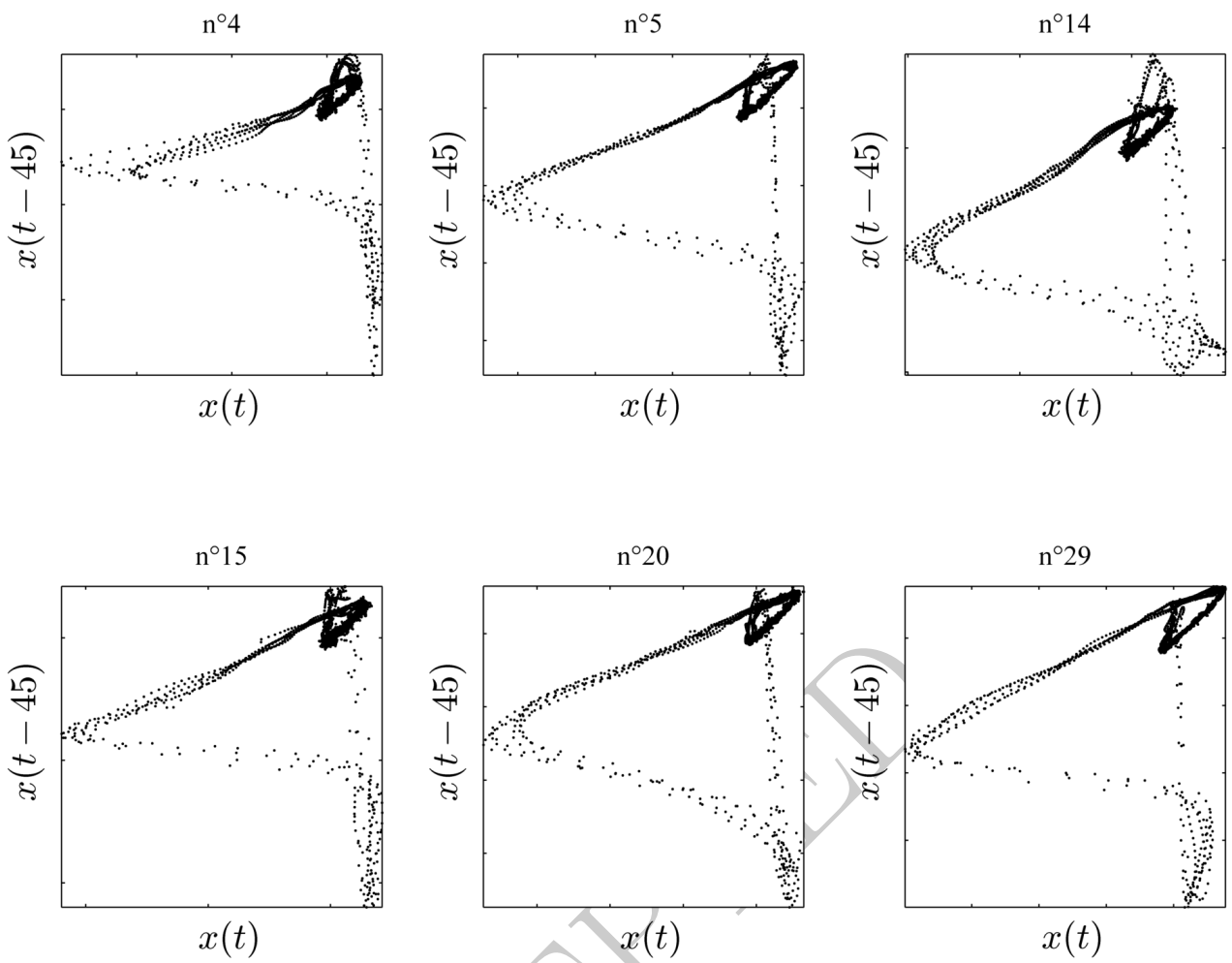

(a)
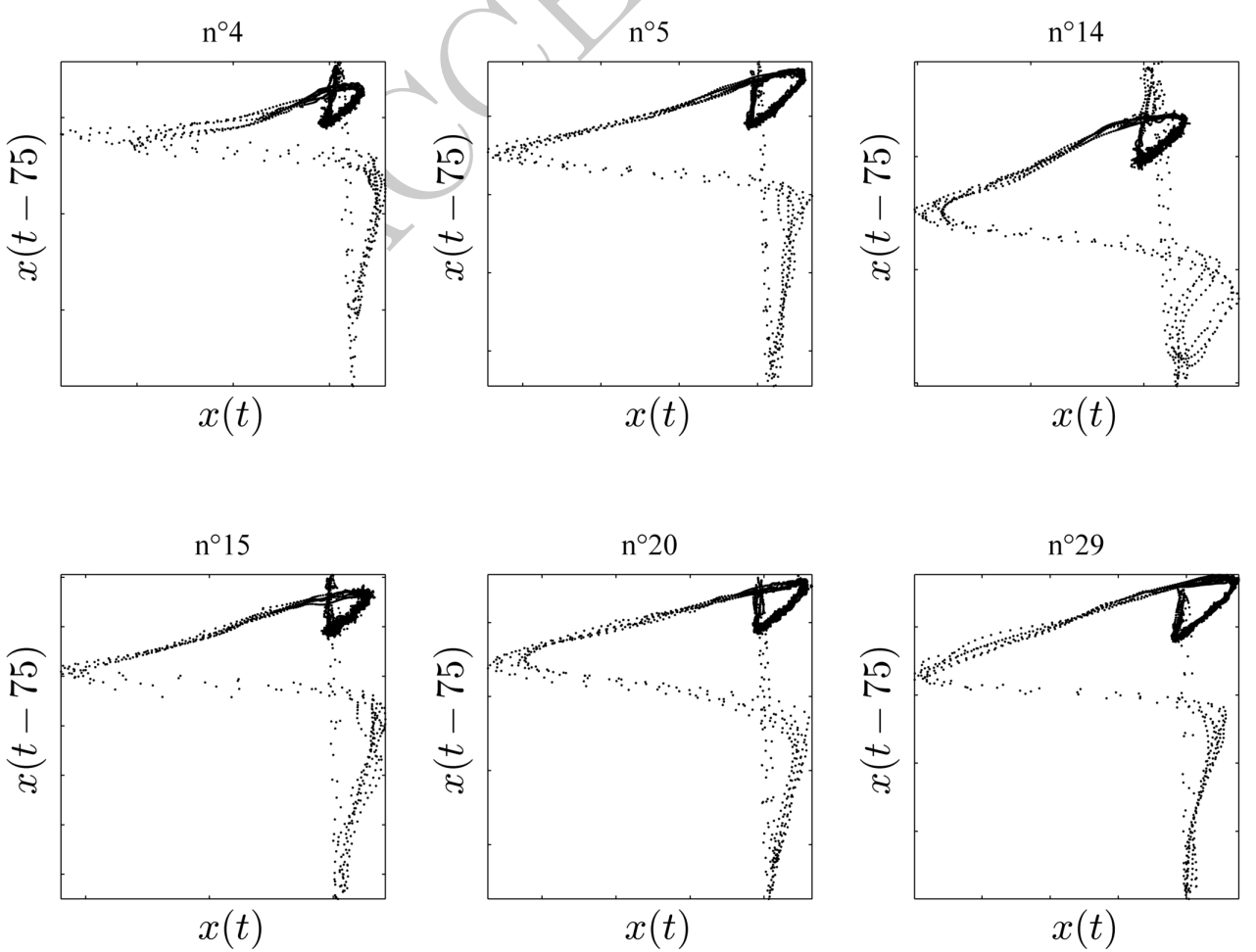

(b)

Figure 11. Examples of 2D projection of the phase space reconstruction for normal signals. The electrode numbers are 4, 5, 14, 15, 20, 29. (a) $\tau=42$ and (b) $\tau=75$. 

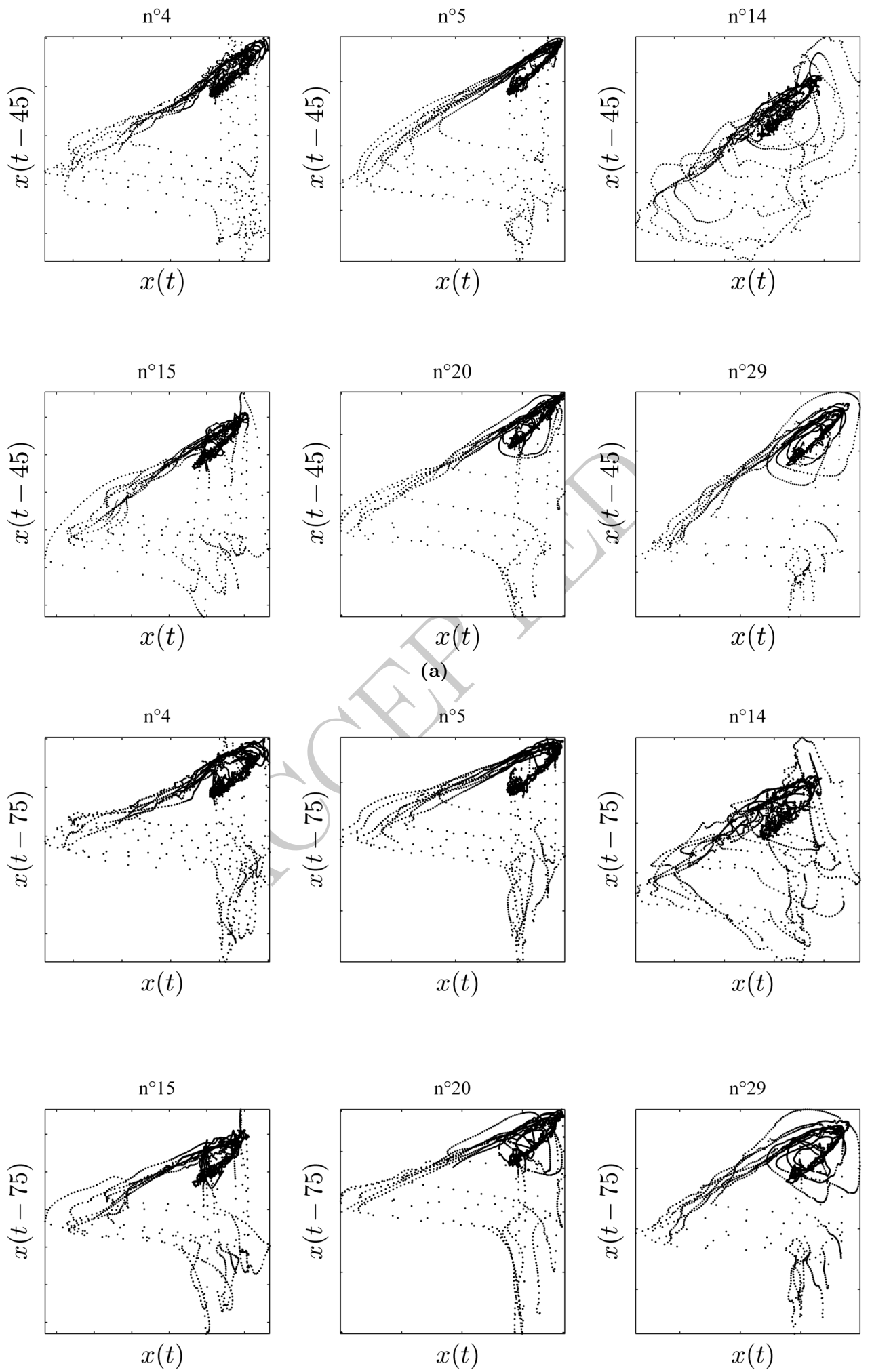

(b)

Figure 12. Example of $2 \mathrm{D}$ projection of the phase space reconstruction for arrhythmic signals. The electrode numbers are 4, 5, 14, 15, 20, 29. (a) $\tau=45$ and (b) $\tau=75$. 

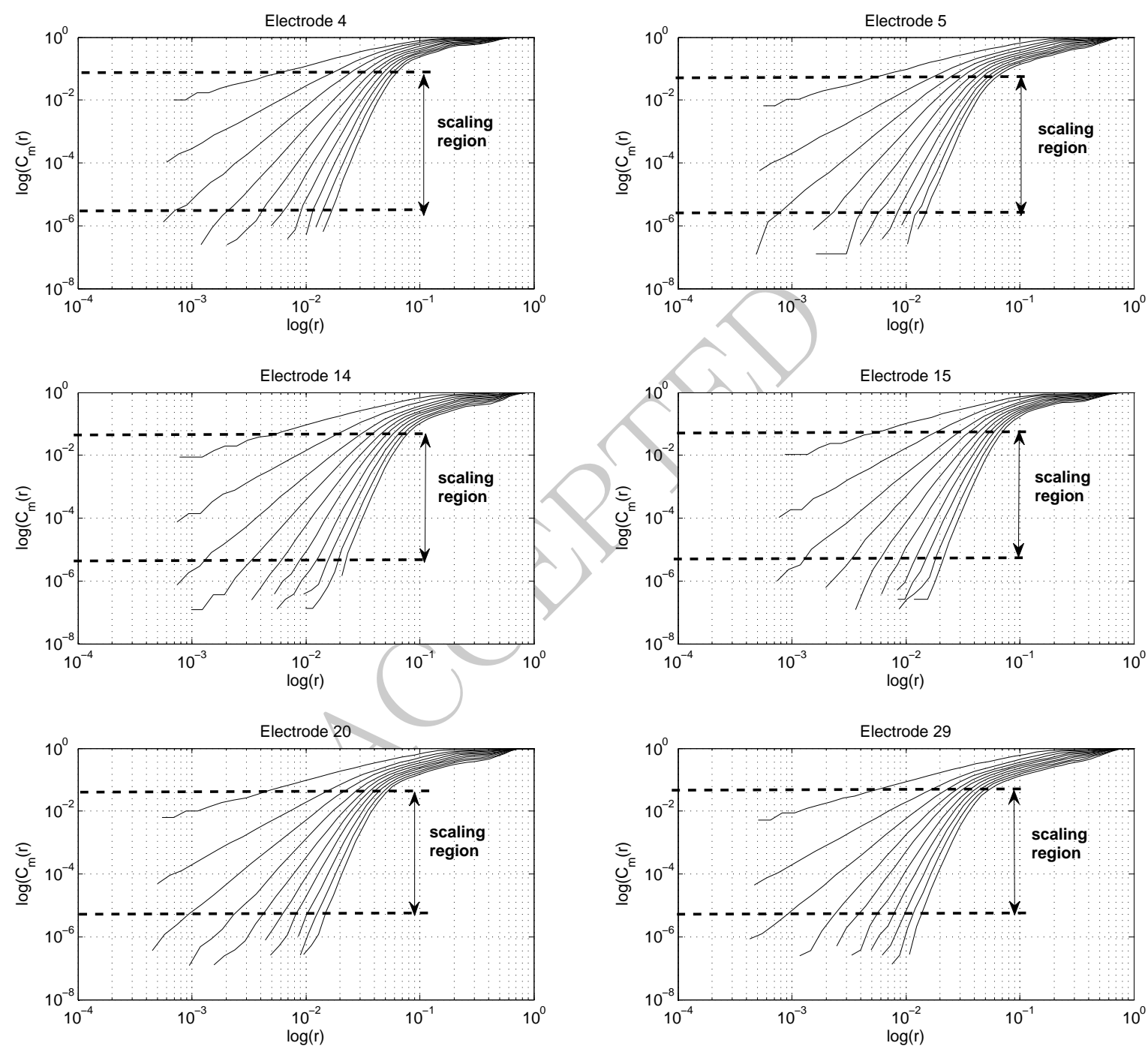

Figure 13. Normal EFP signals : Correlation integral $C_{m(r)}$ from a EFP time series. The correlation integral is plotted at embedding dimension $m=1,2,3,4,5,6,7,8,9,10$ (top to bottom curves). 

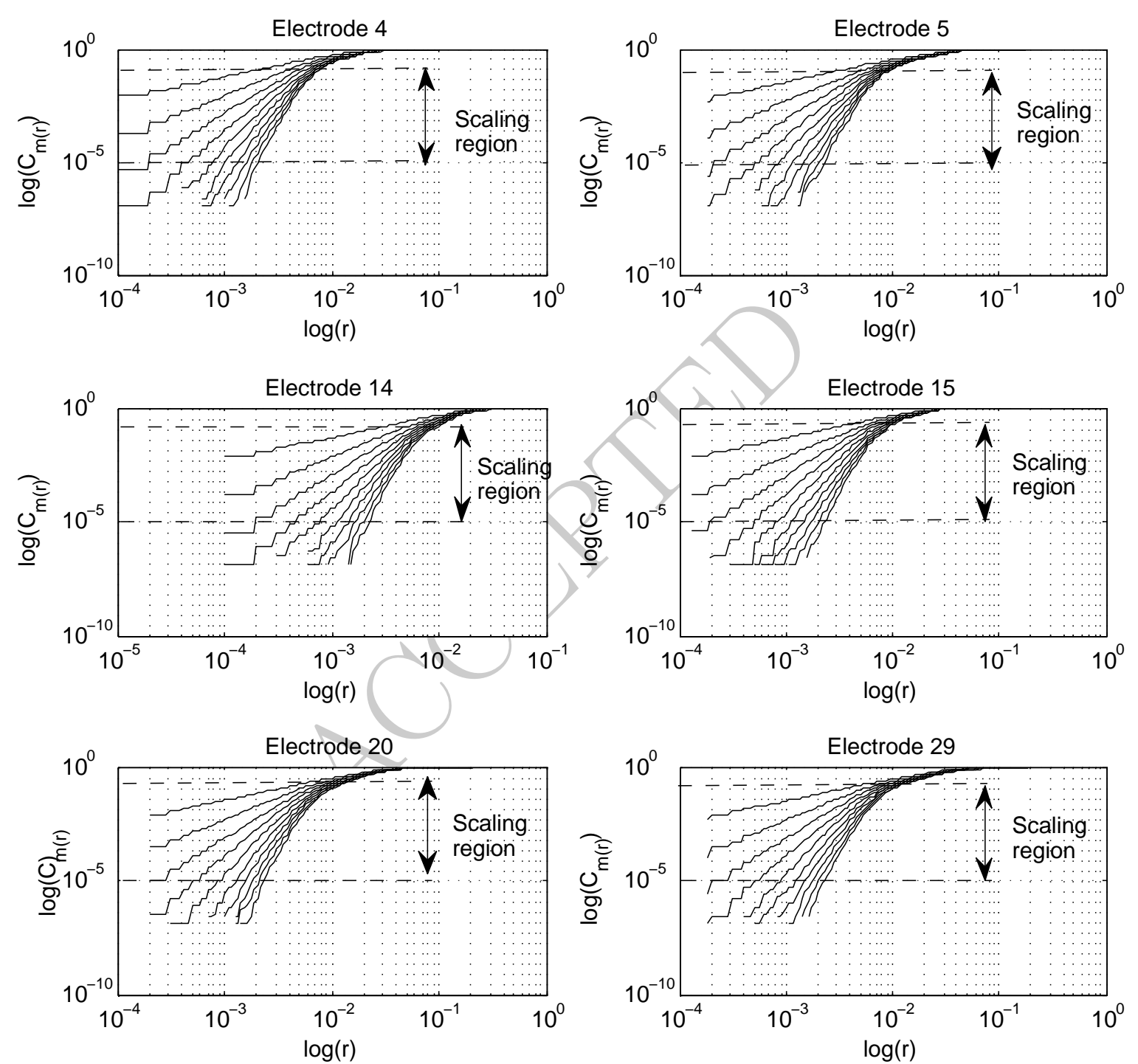

Figure 14. Arrhythmic EFP signals : Correlation integral $C_{m(r)}$ from a EFP time series. The correlation integral is plotted at embedding dimension $m=1,2,3,4,5,6,7,8,9,10$ (top to bottom curves). 

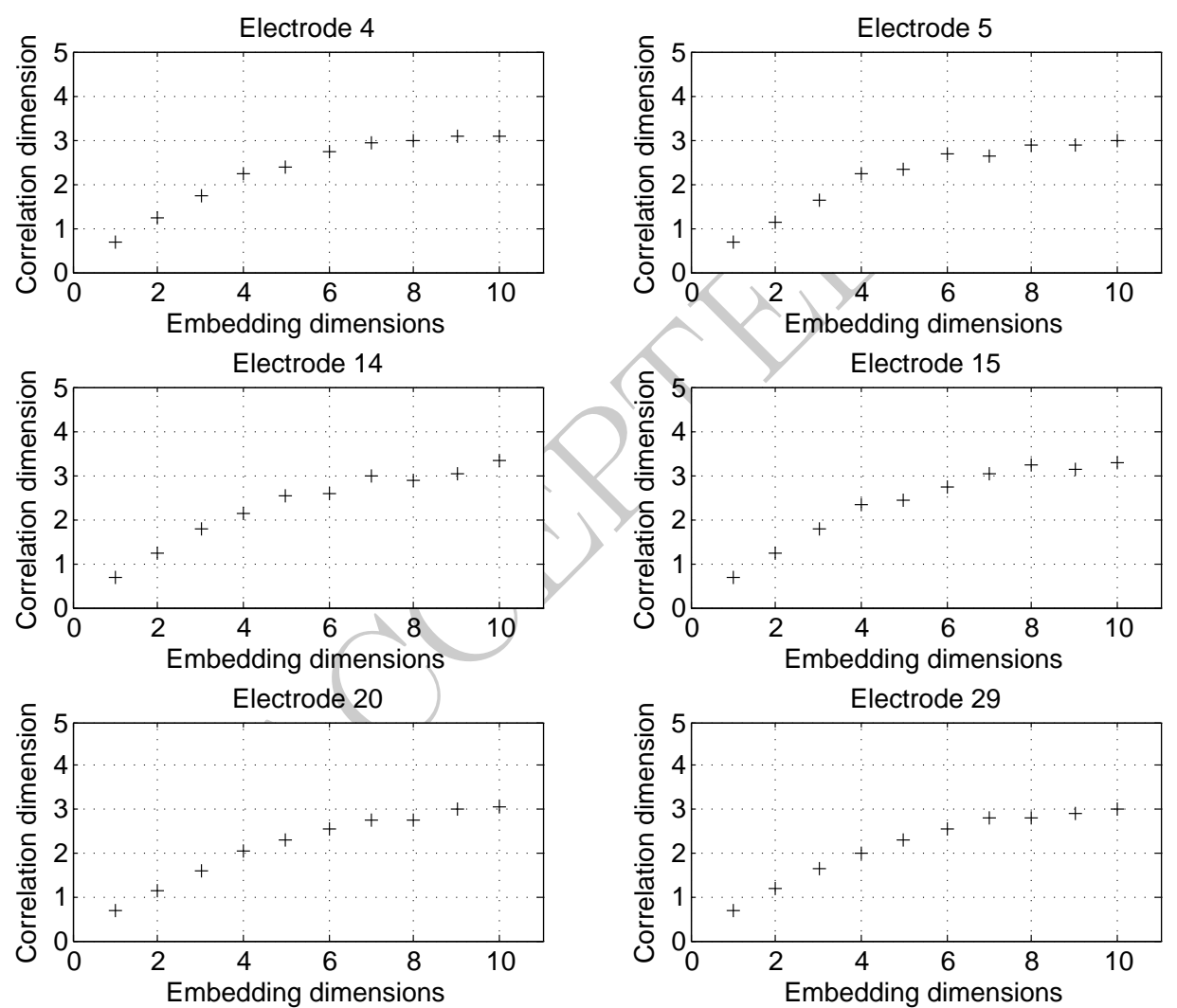

Figure 15. Normal EFP signals : Correlation dimension $D$ function of embedding dimension $m$. 

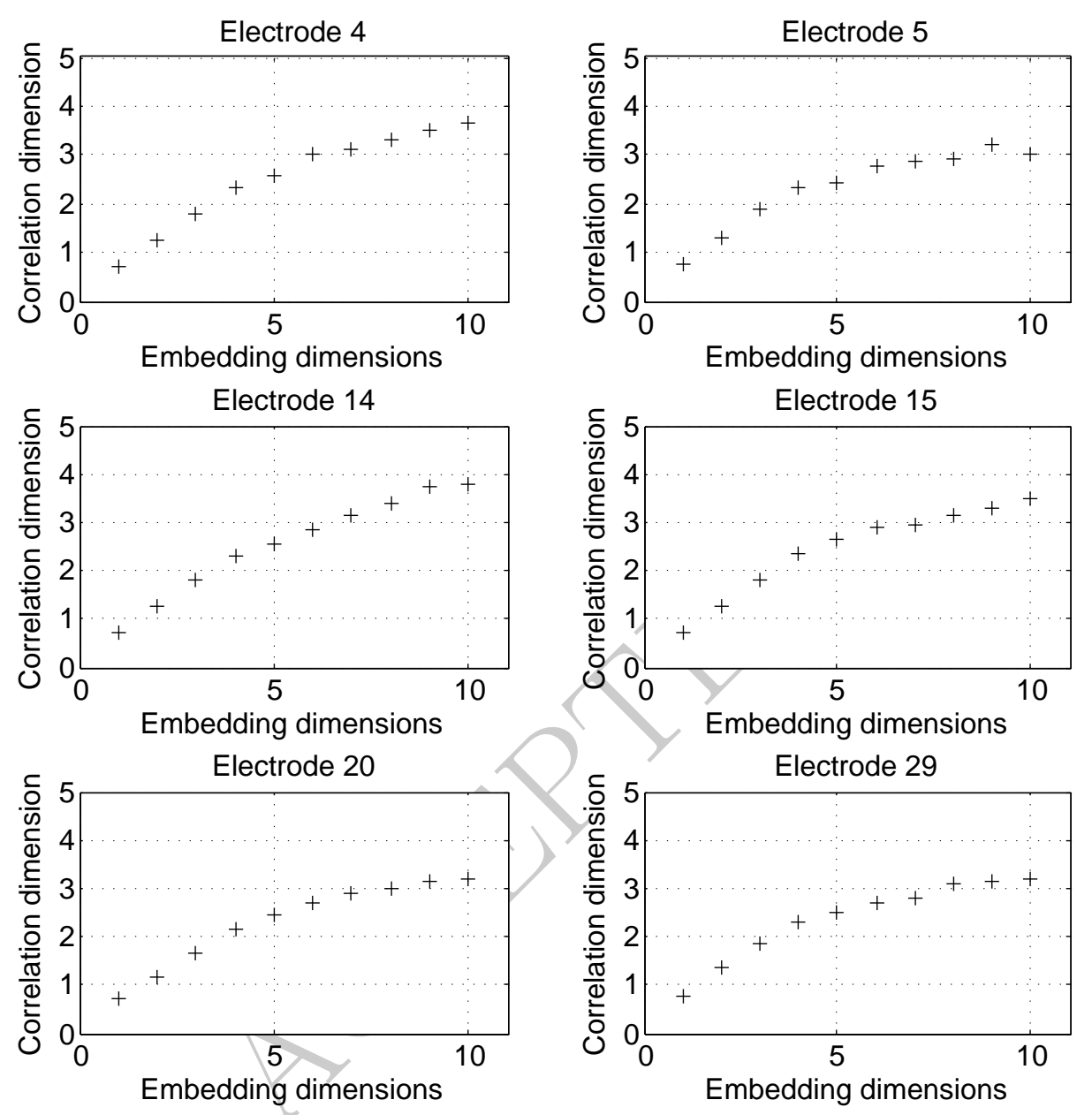

Figure 16. Arrhythmic EFP signals: Correlation dimension $D$ function of embedding dimension $m$.

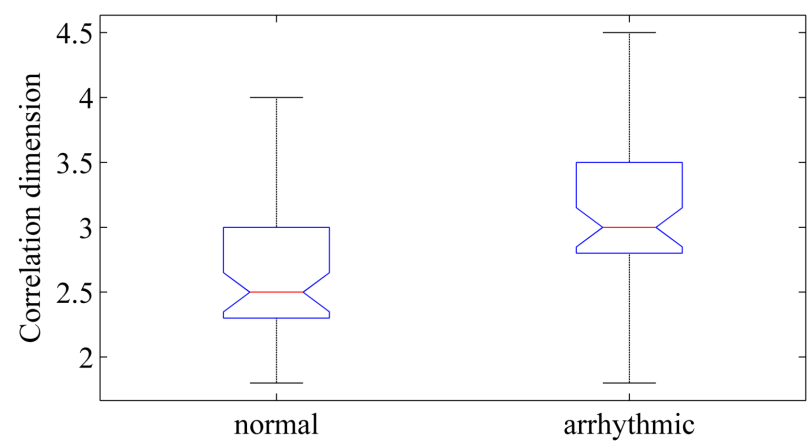

Figure 17. Distribution of Correlation dimension for normal and arrhythmic EFP signals $\left(p=2.774210^{-4}\right.$, Kruskal-Wallis test with $\left.n=52\right)$. 


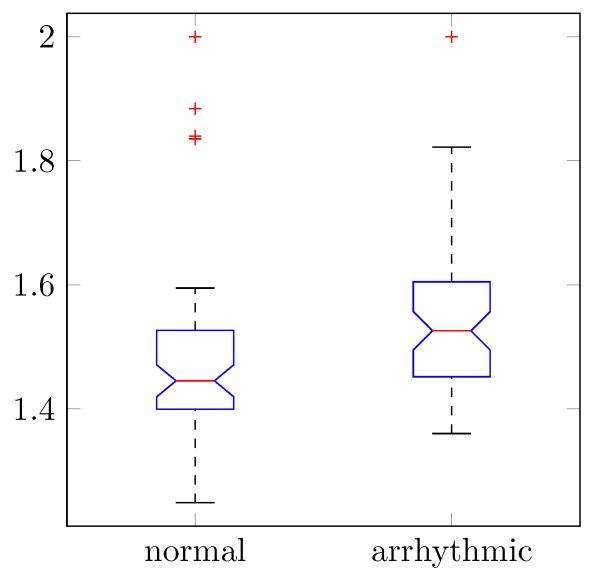

Figure 18. Distribution of Fractal dimension (estimated from Boxcount) for normal and arrhythmic EFP signals $(p=0.0006332$, Kruskal-Wallis test with $n=52)$.

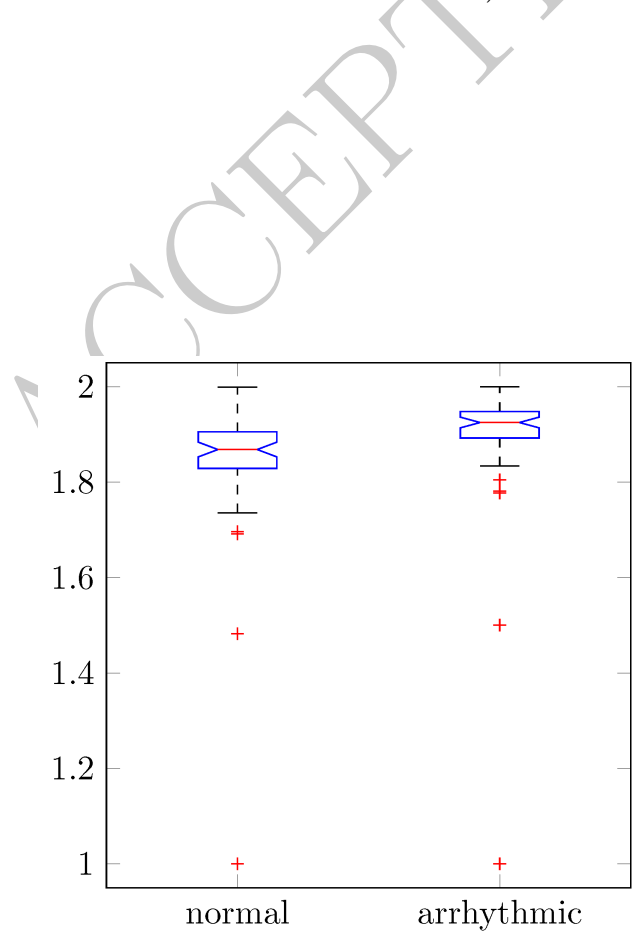

Figure 19. Distribution of Fractal dimension (estimated from Hall-Wood) for normal and arrhythmic EFP signals $\left(p=5.622610^{-6}\right.$, Kruskal-Wallis test with $\left.n=52\right)$. 


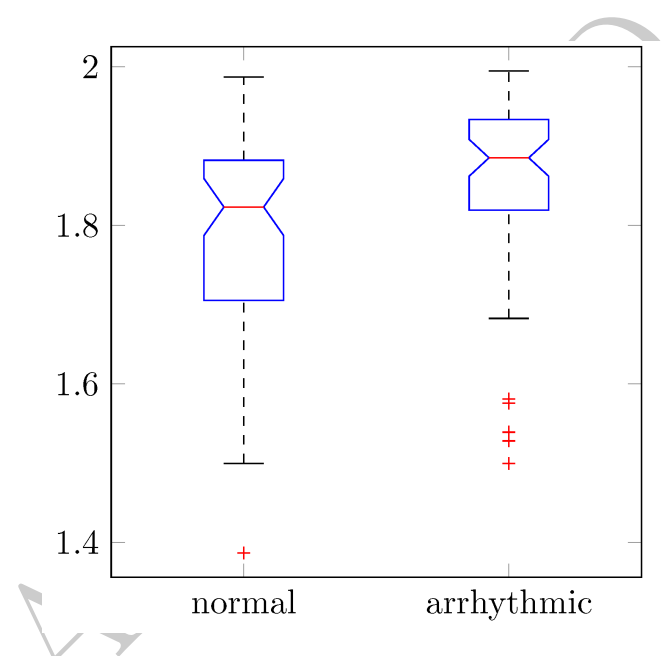

Figure 20. Distribution of Fractal dimension (estimated from Variogram) for normal and arrhythmic EFP signals ( $p=0.0006334$, Kruskal-Wallis test with $n=52$ ). 